\title{
Error-based Structure Prediction in Comprehension: Evidence from verb bias effects in a visual-world structural priming paradigm for Mandarin Chinese
}

\author{
Xuemei Chen ${ }^{1}$, Suiping Wang ${ }^{2,3,4,5}$, and Robert J. Hartsuiker ${ }^{1}$ \\ ${ }^{1}$ Department of Experimental Psychology, Ghent University, Belgium \\ ${ }^{2}$ Key Laboratory of Brain, Cognition and Education Sciences, Ministry of Education, South China \\ Normal University \\ ${ }^{3}$ School of Psychology, South China Normal University \\ ${ }^{4}$ Center for Studies of Psychological Application, South China Normal University \\ ${ }^{5}$ Guangdong Key Laboratory of Mental Health and Cognitive Science, South China Normal University
}

\section{Author Note}

Xuemei Chen ${ }^{\text {iD }}$ htps://orcid.org/0000-0003-0798-8616

Suiping Wang https://orcid.org/0000-0001-8726-169X

Robert J. Hartsuiker (iD) https://orcid.org/0000-0002-3680-6765

We have no known conflict of interest to disclose.

Corresponding authors at: School of Psychology, South China Normal University,

Guangzhou 510631, China (S. Wang). Department of Experimental Psychology, Ghent 9000,

Belgium (X. Chen). E-mail addresses: wangsuiping@m.scnu.edu.cn (S. Wang),

xuemei.chen@ugent.be (X. Chen). ${ }^{1}$

\footnotetext{
1 This paper was accepted by Journal of Experimental Psychology: Learning, Memory, and Cognition. (C) 2021, American Psychological Association. This paper is not the copy of record and may not exactly replicate the final, authoritative version of the article. Please do not copy or cite without authors' permission. The final article will be available, upon publication, via its DOI: 10.1037/xIm0001048
} 


\begin{abstract}
Structural priming studies in production have demonstrated stronger priming effects for unexpected sentence structures (inverse preference effect). This is consistent with error-based implicit learning accounts that assume learning depends on prediction error. Such prediction error can be verb-specific, leading to strong priming when a verb that is for instance biased towards the prepositional object(PO) structure occurs with an unexpected double object(DO) structure. However, it is unclear whether this mechanism also holds for language comprehension, especially for languages like Mandarin Chinese, which arguably depends strongly on semantics to predict syntax in comprehension. Experiment 1 was a norming study $(\mathrm{N}=367)$ that measured the biases (DO vs. PO) of 48 Mandarin Chinese dative verbs. Experiment 2 (N=72) crossed verb bias (DObias or PO-bias) and structure (DO or PO) of prime sentences in a visual-world paradigm, to examine whether Mandarin comprehenders show an inverse preference effect. The priming effect is expressed as the proportion of looks to the predicted referent (i.e., the recipient after a DO-prime, the theme after a PO-prime), for two critical time windows during target sentence processing: the verb and the first syllable of the first post-verbal noun (which was identical in theme and recipient). There was priming in both time windows, even though the verb differed between prime and target. Importantly, there was an inverse preference effect (i.e., stronger priming after a DO prime with a PO-biased verb than with a DO-biased verb) in the second time window. These results provide evidence for an error-based structure prediction system in comprehension.
\end{abstract}

Key words: Implicit learning; verb bias; structural priming; comprehension; Mandarin Chinese 


\section{Introduction}

In order to efficiently process a sentence during language comprehension, people make predictions from previous experience (e.g., "it was a windy day so the boy went to the park to fly a kite") to overcome the noise of variable input (Levy, 2008). They will predict many aspects of the upcoming words, including semantic (e.g., "kite" is inanimate) and syntactic (e.g., word category, "kite" is a noun) information. Moreover, they will also predict larger units of syntax, such as the structure of a verb phrase (e.g., dative structure, double object (DO) "show the horse the book" or prepositional object (PO) "show the horn to the dog") (Delong et al., 2014; Thothathiri \& Snedeker, 2008a). But it is still unclear what mechanism generates these predictions of structure in language processing. In this study, we investigated the predictions of an implicit learning account, which assumes an error-based prediction system. Specifically, because comprehenders aim to minimize prediction error during language processing, they rapidly adapt upon experiencing prediction error and thereby continuously renew the syntactic system that generated the structure prediction (Chang, 2000; Chang et al., 2006). We will test this model's prediction that encountering a verb together with an unexpected structure (hence large prediction error) results in relatively large structural priming in comprehension.

Structural priming is the tendency for speakers and comprehenders to re-produce or predict the syntactic structures of a recently processed prime sentence in the target sentence (Bock, 1986b, 1986a; Mahowald et al., 2016; Pickering \& Branigan, 1998). For example, speakers tend to produce DO sentences more often after processing DO sentences than after PO sentences (Bock, 1986b). Importantly, structural priming is influenced by the structural preference of individual verbs (so-called verb bias). Jaeger and Snider (2013) calculated the bias for the alternative structure of each prime verb (Jaeger \& Snider, 2008), based on a corpus of speech production (Bresnan et al., 2007). For example, speakers prefer to follow the English verb "show" with the DO structure rather than the PO structure. Because of the existing bias towards DO, the verb "show" followed by a PO structure in the prime leads to a substantial prediction error. The degree of prediction error depends on the extent to which a given verb biases towards the specific structure. Jaeger and Snider found that when the prime verb was followed by a more surprising structure (hence larger prediction error), speakers were more likely to reuse the structure from the primes, in both spoken and written production of target sentences, indicating a cross-modality inverse preference priming effect. Similar results were found in Dutch, when verb bias was calculated from a picture 
description task with specific verbs (Bernolet \& Hartsuiker, 2010). Additionally, the inverse preference priming effect was found in two age groups of children (3-4 or 5-6 years old) and adults (Peter et al., 2015). This inverse preference priming effect supports an important assumption of implicit learning accounts, namely that the acquisition of syntax is based on the prediction error for the upcoming word (Chang et al., 2006). This account claims that when the prediction does not match the actual input, like PO after "show", the increased error will strengthen the connection weights of the units that represent the experienced structure. These weight changes then lead to increased use of this experienced structure. Therefore, this account claims that structural priming is a form of implicit learning, and that it is driven by prediction errors after recent exposure as the system aims to decrease future prediction errors (Bock \& Griffin, 2000; Chang, 2000; Chang et al., 2006).

The inverse preference effect in language production thus supports an error-based prediction account (Chang et al., 2006). According to this account, the same error-based prediction mechanism is responsible for structural priming in comprehension as well. However, in contrast to the robust finding of stable structural priming when the verb differs between prime and target in production, only some comprehension studies showed such abstract structural priming (Thothathiri \& Snedeker, 2008a, 2008b; Traxler, 2008), whereas other studies did not (Arai et al., 2007; Branigan et al., 2005; Ziegler \& Snedeker, 2019). For instance, Arai et al. (2007) observed structural priming when the verb was repeated between prime and target in a visual world paradigm. Specifically, participants looked more often at the animate recipient (predicting DO structure) than at the inanimate theme after the processing of DO primes, when they heard the target verbs; they looked more at the theme (predicting PO structure) than recipient after PO primes. However, such priming disappeared when the verb differed from prime and target, suggesting a lexically dependent priming effect in comprehension. In contrast, Thothathiri and Snedeker (2008a) reported evidence for lexically independent priming. These authors used an act-out task that made the post-verbal arguments after the target verb more unpredictable than in Arai et al.'s study. Moreover, there was initial phonemic overlap between recipients and themes in target sentences (e.g., "horse" vs. "horn") and there were two primes with the same structure, embedded in a story context, before the target sentences. There was structural priming in the time window of the first noun phrase (e.g., "hor...") for both three- and four-year-old children and adults even though the verbs were different (Thothathiri \& Snedeker, 2008a, 2008b). The presence of such lexically 
independent priming, similar to the findings in production, supports the hypothesis of a shared syntactic processing mechanism in production and comprehension (Traxler, 2008).

Is this lexically independent structural priming in comprehension induced by an errorbased prediction mechanism? One way of testing this is to assess the inverse preference priming effect in comprehension, similar to the studies in production. Fine and Jaeger (2013) addressed this issue by re-analyzing the eye-tracking data of Thothathiri and Snedeker (2008a) to investigate whether priming in comprehension depended on prediction errors in primes. To estimate prediction error, they performed a sentence-completion task and then calculated the alternation structure bias (the probability of DO vs. PO responses) for each of Thothathiri and Snedeker's prime sentences. There was an interaction between the structure bias of the first prime sentences (not for the second prime sentences) and prime structure (DO vs. PO) such that priming was stronger when prime structure was more surprising. The findings are consistent with the results of production studies (Jaeger \& Snider, 2013). However, Fine and Jaeger's study was not an experimental study but a re-analysis of an existing experiment that did not originally control structure bias and that was not set up to address the question of error-based prediction.

Below, we report a controlled experiment that tests whether there is an error-based structure prediction mechanism in comprehension, which is responsible for both lexically independent structural priming and inverse preference priming. Such a test is important, because: 1) previous evidence for lexically independent structural priming in comprehension is mixed;2) it is not clear whether such evidence is based on the specifics of the task (e.g., having two primes rather than one); 3) there is no experimental evidence for an inverse preference effect in comprehension (only a reanalysis of an experiment not designed to address that question); 4) the existing evidence is restricted to only a single language (English).

Our study therefore adds to the existing literature in at least two important ways. First, we manipulated verb bias and prime structure experimentally in a visual world paradigm. In contrast, the experiment that Fine and Jaeger (2013) reanalyzed did not strictly manipulate verb bias and used two prime sentences before each target sentence. The repeated exposure of unexpected structure might have led to adaptation and so decreased the effect of prediction error, which might be the reason that there was no inverse preference priming with the second prime sentences. Therefore, we only presented one prime sentence before the target sentences. If structure prediction 
depends on the error-based mechanism, we expect an interaction between structural priming and verb bias.

Second, we investigated structure prediction in the comprehension of Mandarin Chinese, a language with flexible word order and few explicit markers of syntactic structure. In particular, Mandarin lacks morphophonological markers like case, tense, grammatical gender, and number, which many languages use as direct cues to distinguish the grammatical roles of the sentence constituents. This results in a difficulty to detect syntactic information (i.e., category) of individual words. A further source of potential difficulty is that Mandarin allows various word orders (i.e., SVO, OSV, SOV, VOS) (P. Li, 1996; P. Li et al., 1993; L. Zhang, 1997). These properties might result in a difficulty to predict or pre-access structure information independently before access of the target verb in the bottom-up processing of comprehension. Indeed, many Mandarin comprehension studies proposed that semantic processing precedes syntactic processing (Wang et al., 2013; Yang et al., 2009; Y. Zhang et al., 2013). However, structure prediction may involve abstract structural knowledge rather than lexically dependent knowledge (Perek \& Goldberg, 2015, 2017; Thothathiri \& Braiuca, 2020; Thothathiri \& Rattinger, 2016). If an error-based prediction mechanism involving abstract structure representation holds for all languages, we expect structural priming in Mandarin, similarly to English.

In the eye-tracking experiment reported below we needed to strictly control the level of verb bias in prime and target. Therefore, we normed a large number of Mandarin dative verbs in Experiment 1 for verb bias. Earlier studies on structural priming in Mandarin showed a preference for the PO structure over the DO structure in production (Z. Cai et al., 2011, 2012, 2015; Chen et al., 2019; Huang et al., 2016, 2019). We therefore expected to find a PO-bias overall, but possibly with considerable variation among dative verbs. Then in Experiment 2, we investigated inverse preference priming within a visual world paradigm (Arai et al., 2007). In the paradigm, participants first read aloud written prime sentences and then listened to spoken target sentences while looking at pictures with corresponding objects, allowing us to detect syntax-based predictions in real time (Huettig et al., 2011; Tanenhaus et al., 1995). We manipulated the verb bias (DO-bias vs. PO-bias) and structure type (DO vs. PO) in the prime sentences. The target verbs differed from prime verbs and had no obvious preference for DO or PO. If comprehension involves a lexically independent representation of syntax in Mandarin, structural priming should occur even when the verb is not repeated between the prime and target. Importantly, if comprehension involves error-based 
prediction, structural priming should interact with the structural bias of the specific verb, so that priming is stronger when verb bias mismatches prime structure.

\section{Experiment 1: norming study of verb bias in Mandarin}

\section{Method}

Participants

We recruited 404 native Mandarin speakers for the online tests. The tests were shared on several social platforms (e.g., Wechat or QQ) to reach our target group of university students from China. They used Mandarin as a dominant language in their daily life. We excluded 37 participants who did not follow the instruction to describe the target pictures (i.e., writing numbers or other content unrelated to the target pictures), leaving a final sample of 367 participants. Most participants (75\%) came from Guangdong Province and the rest (25\%) came from the other 19 Provinces or Municipalities of China.

\section{Materials}

We selected 48 single-character dative verbs from the corpus SUBTLEX-CH (Chinese Word and Character Frequencies; Cai \& Brysbaert, 2010). The alternation verb bias, DO vs. PO, was measured in a picture description task (Bernolet \& Hartsuiker, 2010). For each verb, three entities were used to construct a picture of a ditransitive event involving an agent, recipient, and theme. There were two versions of these pictures with different positions of recipient and theme (see Figure 1; these were the same materials as used by Huang et al., 2016). Similar to Arai et al. (2007), the three entities did not express an action (i.e., they were static), allowing participants to construct the verb-relating event flexibly. These 48 verbs were divided into 2 parts, so that there were 24 verbs for each version of the test and 2 versions for each verb. Thus, we had four versions of the online test.

\section{Figure 1}

Example of picture description task in the testing of verb bias 

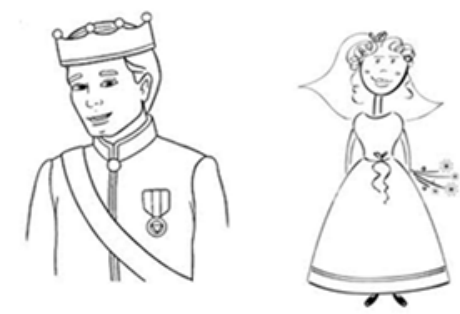

送

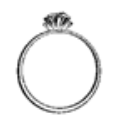

Version A
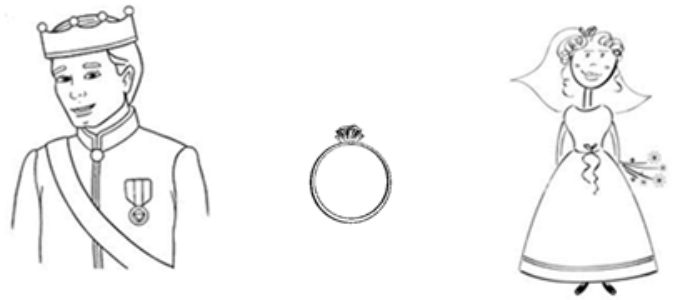

送

Version B

Note. "Version A" on the left presents the theme on the right, and the potential agent or recipient on the left and in the middle. "Version B" presents the theme in the middle, and the potential agent and recipient on the left or right. The verb underneath the three entities "送” means "give".

\section{Procedure}

Participants were instructed to describe the picture with one sentence in Mandarin using the provided verb (Bernolet \& Hartsuiker, 2010). Responses were provided in the written modality. The test took around 10 minutes.

Scoring

We coded all responses as DO, PO, or others. If a verb was followed by the recipient and then the theme, we coded it as DO. If a verb was followed by the theme and then a preposition and recipient, we coded it as PO. Otherwise, we coded the responses as others.

\section{Results}

We collected 8564 valid responses for the 48 items, with 846 responses coded as DO (9.88\%), 1791 responses coded as PO (20.91\%), and 5927 responses coded as others (69.21\%). The verb bias was computed as the log-odds for the valid response of DO or PO structure (Bernolet \& Hartsuiker, 2010; Jaeger \& Snider, 2008). A negative value indicates the verb has a PO-bias and a positive value indicates it has a DO-bias. These 48 verbs showed a strong preference for the PO structure on average $($ Mean $=-1.47, \mathrm{SD}=1.34$ ) (see Figure 2$)$. Over $85 \%$ of verbs were PO-biased, 8 verbs were extremely PO-biased without any DO responses, and only 7 verbs were DO-biased with slightly more DO than PO responses. Based on this distribution of verb bias in Mandarin, we 
chose four DO-biased verbs (Mean=0.92, $\mathrm{SD}=0.40$ ) and four PO-biased verbs (Mean=-2.01, $\mathrm{SD}=0.34$ ) for the prime sentences, and four neutral-biased (slightly PO-biased) verbs (Mean=-0.22, $\mathrm{SD}=0.12$ ) for the target sentences (see Table 1). It is important to note that even though we chose verbs that were strongly biased (especially the PO-biased verbs), each verb can in principle occur with both DOs and POs and that indeed, both DOs and POs were attested in our data set for each selected verb.

Due to the flexibility of description for the target pictures (i.e., participants were not instructed to use the classical ditransitive structures (DO or PO) to describe the pictures), we found a high proportion of other responses, which is similar to the corpus data of Dutch newspapers (e.g., 74.7\% other responses, see Colleman, 2006, 2009; Colleman \& Bernolet, 2013). The second reason for the high proportion of other responses is that Mandarin dative verbs have more than two structural options to describe the target pictures. For example, these other responses involved shifted dative structures (e.g., shifted PO, "wangzi gei xinniang song le jiezhi", "the prince to the bride gives a ring"; or BA-DO, "wangzi ba jiezhi song geile xinniang", "the prince BA a ring gives the bride"), active or passive sentences, and even relative clauses. These responses may result from the flexible word order of Mandarin Chinese (C. N. Li \& Thompson, 1989; P. Li, 1996; P. Li et al., 1993; W. Zhang, 1991). However, the basic word order of dative structures in Mandarin Chinese is still "[V NP NP]" or "[V NP PP]", which differs from shifted dative structures (Man, 2003). Thus, we coded the latter responses as other responses.

\section{Figure 2}

Distribution of the structure bias of 48 dative verbs in Mandarin 


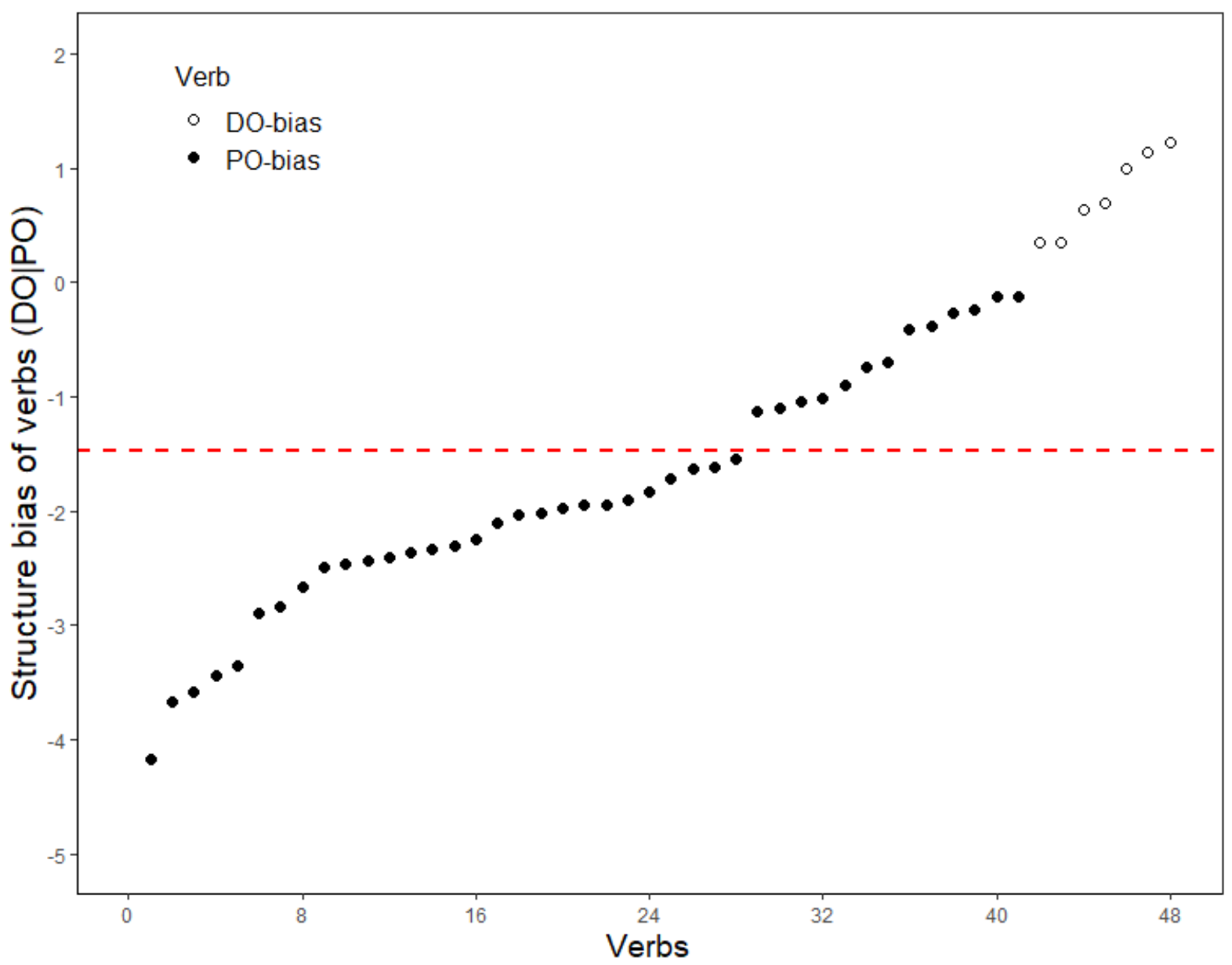

Note. Structural bias of verbs was calculated as the logarithm of the frequency of the DO structure following the verb divided by the frequency of the alternative PO structure (i.e., " $\log [(\# \mathrm{DO}+1) /(\# \mathrm{PO}+1)]$ ", with 1 added to both operands to avoid division by zero). Values larger than 0 indicate a DO-bias (i.e., white circle) and values below 0 indicate a PO-bias (i.e., black circle). The red line in the middle represents the mean value (-1.47) of all 48 dative verbs.

\section{Table 1}

Frequency of DO and PO responses and alternation bias of 48 dative verbs.

\begin{tabular}{|c|c|c|c|c|c|c|c|c|c|}
\hline \multirow{2}{*}{ Verb } & \multicolumn{3}{|c|}{ Response frequencies } & \multirow{2}{*}{$\begin{array}{l}\text { Verb } \\
\text { bias }\end{array}$} & \multirow{2}{*}{ Verb } & \multicolumn{3}{|c|}{ Response frequencies } & \multirow{2}{*}{$\begin{array}{l}\text { Verb } \\
\text { bias }\end{array}$} \\
\hline & DO & $P O$ & Others & & & $D O$ & $P O$ & Others & \\
\hline 做(make) & 0 & 64 & 115 & -4.17 & 派(send) & 4 & 27 & 146 & -1.72 \\
\hline 抓(catch) & 0 & 38 & 137 & -3.66 & 捎(bring along) & 10 & 55 & 110 & -1.63 \\
\hline 捉(catch) & 0 & 35 & 144 & -3.58 & 留(leave) & 8 & 44 & 131 & -1.61 \\
\hline 捡(pick up) & 0 & 30 & 147 & -3.43 & 汇(transfer) & 11 & 55 & 114 & -1.54 \\
\hline
\end{tabular}


VERB BIAS IN MANDARIN COMPREHENSION

$\begin{array}{lccccccccc}\text { 摘(pick) } & 1 & 56 & 123 & -3.35 & \text { 寄(post) } & 17 & 55 & 103 & -1.13 \\ \text { 找(find) } & 0 & 17 & 166 & -2.89 & \text { 扔(throw) } & 10 & 32 & 136 & -1.10 \\ \text { 提(carry) } & 2 & 50 & 125 & -2.83 & \text { 租(rent) } & 6 & 19 & 154 & -1.05 \\ \text { 拉(drag) } & 2 & 42 & 134 & -2.66 & \text { 分(divide) } & 19 & 54 & 101 & -1.01 \\ \text { 抬(lift) } & 0 & 11 & 167 & -2.48 & \text { 卖(sell) } & 14 & 36 & 128 & -0.90 \\ \text { 投(throw) } & 2 & 34 & 141 & -2.46 & \text { 抛(throw) } & 26 & 56 & 98 & -0.75 \\ \text { 丢(throw) } & 2 & 33 & 140 & -2.43 & \text { 让(offer) } & 15 & 31 & 132 & -0.69 \\ \text { 倒(pour) } & 1 & 21 & 156 & -2.40 & \text { 借(borrow) } & 19 & 29 & 134 & -0.41 \\ \text { 捧(hold) } & 2 & 31 & 149 & -2.37 & \text { 递(pass) } & 33 & 49 & 102 & -0.39 \\ \text { 买(buy) } & 3 & 40 & 135 & -2.33 & \text { 配(assign) } & 18 & 24 & 140 & -0.27 \\ \text { 拣(pick up) } & 2 & 29 & 150 & -2.30 & \text { 赠(gift) } & 46 & 59 & 72 & -0.24 \\ \text { 拿(take) } & 7 & 75 & 94 & -2.25 & \text { 还(return) } & 43 & 49 & 86 & -0.13 \\ \text { 捐(donate) } & 8 & 73 & 97 & -2.11 & \text { 赔(compensate) } & 53 & 60 & 69 & -0.12 \\ \text { 转(transfer) } & 5 & 45 & 129 & -2.04 & \text { 送(give) } & 82 & 58 & 38 & 0.34 \\ \text { 写(write) } & 3 & 29 & 145 & -2.01 & \text { 交(hand) } & 33 & 23 & 121 & 0.35 \\ \text { 订(order) } & 3 & 28 & 147 & -1.98 & \text { 收(receive) } & 14 & 7 & 156 & 0.63 \\ \text { 搬(move) } & 0 & 6 & 172 & -1.95 & \text { 抢(rob) } & 7 & 3 & 165 & 0.69 \\ \text { 叫(order) } & 0 & 6 & 171 & -1.95 & \text { 赏(grant) } & 107 & 39 & 32 & 0.99 \\ \text { 退(refund) } & 3 & 26 & 149 & -1.91 & \text { 授(reward) } & 83 & 26 & 70 & 1.13 \\ \text { 发(send) } & 7 & 49 & 122 & -1.83 & \text { 瑒(award) } & 115 & 33 & 34 & 1.23\end{array}$

Note. The verbs in italic were chosen as the prime or target verbs in Experiment 2. PO-biased prime verbs included “丢(throw)”, “捐(donate)”, “退(refund)” and “留(leave)”. DO-biased prime verbs included “送(give)”, “赏(grant)”, “授(reward)” and “赐(award)”. Neutral-biased target verbs included “递(pass)”, “赠(gift)”, “还(return)” and “赔 (compensate)".

\section{Discussion}

We observed a strong overall PO structure preference in Mandarin Chinese (i.e., 846 DO vs. $1791 \mathrm{PO}$ responses, thus 68\% PO responses overall). This PO-bias is comparable to that observed in the intransitive baseline conditions of Mandarin structural priming in production experiments (i.e., 65\% in Z. Cai et al., 2012; 70\% in Chen et al., 2019). Such production experiments were highly constrained to elicit dative responses with a preamble and a target picture 
(e.g., "The policeman gave") and therefore there were hardly any other responses. The alternation biases obtained here, in contrast, reflect Mandarin dative structural preferences in more spontaneous production.

In the experiment below, we presented selected verbs with either a DO- or PO-bias, in order to test whether: 1) there is abstract structural priming in Mandarin sentence comprehension; 2) this priming is sensitive to prediction error.

\section{Experiment 2: inverse preference priming in comprehension}

\section{Method}

Participants

We paid 72 native Mandarin speakers 10 euros to participate in Experiment 2. They were postgraduate students or postdoctoral researchers at Ghent University from all over China. All reported to have normal or corrected-to-normal vision. The study was approved by the ethics committee of the Faculty of Psychology and Educational Sciences, Ghent University.

\section{Materials}

We constructed 48 sets of materials (see https://osf.io/6kcyz/ for the full list). Each set included four prime sentences (DO-biased DO, DO-biased PO, PO-biased DO, and PO-biased PO, see Table 2), and two ditransitive target sentences (PO and DO) with neutral-biased verbs. We chose the theme and recipient nouns in the target sentences so that there was a temporary ambiguity in the first syllable of the first noun phrase (NP1) (e.g., "Qiuyuan [football player]" and "Qiupai [racket]", see Table 2; this was the same manipulation as used by Branigan et al., 2018). Therefore, the sentence remained ambiguous between a DO and PO from the onset of the target verb to the onset of the second syllable of NP1.

\section{Table 2}

Example of one set materials in Experiment 2

Conditions $\quad$ Example

Prime sentences 
a. DO-biased DO

b. DO-biased PO

c. PO-biased DO

d. PO-biased PO

Target sentences

DO

$\mathrm{PO}$
Wujing song Tegong yiba shouqiang.

The policeman gives the spy a gun.

Wujing song shouqiang gei Tegong.

The policeman gives a gun to the spy.

Wujing diu Tegong yiba shouqiang.

The policeman throws the spy a gun.

Wujing diu shouqiang gei Tegong.

The policeman throws a gun to the spy.

Yeye huan Qiuyuan yifu Qiupai.

The grandpa returns the football player a racket.

Yeye huan Qiupai gei Qiuyuan,

The grandpa returns a racket to the football player.

We then divided the 48 sets of materials into 8 lists in a Latin Square design. Each list included 12 prime sentences in each of the four prime conditions. Half of the target sentences had a DO structure and the other half a PO structure, which were counterbalanced for each prime sentence. Additionally, there were three versions of each list to balance orders of the positions of the entities in the target pictures. This was done to balance out effects of spatial expectations of upcoming words (see Figure 3). Additionally, we constructed 96 fillers with a range of sentence structures (e.g., active, passive, shifted-PO, Ba-construction). Half of the fillers also contained homophonic syllables in audio sentences. One half of the fillers had the same structure for the pair of visual and audio sentences and the other half did not. In order to cover up the purpose of this priming study, one third of the filler trials required participants to recall the pictures that they saw in the previous screen and describe the pictures with their own words. To make this cover task more challenging, half of the filler pictures involved one entity that was incongruent with the corresponding audio sentences, and the other half did not. All 144 trials were presented in a 
pseudo-random order.

\section{Figure 3}

Example target picture in Experiment 2
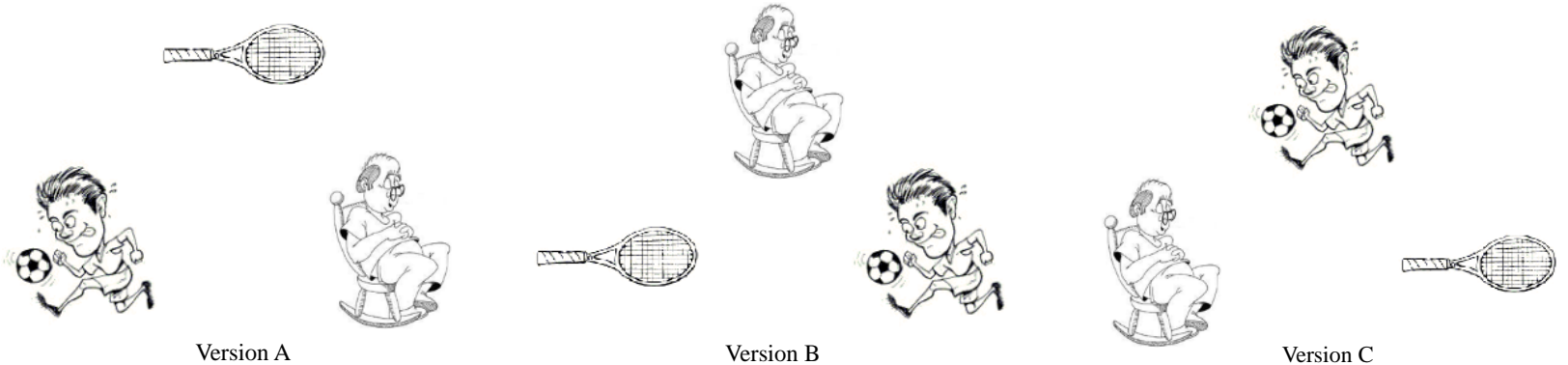

Note. Participants heard DO or PO target sentences (see Table 2) while they saw the corresponding target picture. Version A on the left presents the theme ("racket") on the top, the recipient ("football player") on the left, and the agent ("grandpa") on the right; so the order from top to left to right was "T-R-A". Version B in the middle represents the order "A-T-R" and Version C on the right presents the order "R-A-T".

\section{Procedure}

We used the visual world and structural priming paradigm (Arai et al., 2007). Before the experiment, participants were required to memorize all the entities that were used in the targets and they only began the experiment after they correctly named these entities. During the experiment, participants were instructed to focus on the fixation point so that a drift-correction was triggered. Next, participants read the prime sentence aloud and then pressed the spacebar to trigger the corresponding target picture. They had $1500 \mathrm{~ms}$ to preview the picture. Then, they listened to the audio sentence carefully and watched the target picture at the same time. If they saw 'recall' after target picture presentation (i.e., on $1 / 3$ of the filler trials), they needed to describe the target picture correctly with their own sentence (but note that they mostly repeated the sentences that they just heard verbatim, or in the case of pictures with one mismatching entity, near-verbatim). We recorded the right eye of participants from onset to $350 \mathrm{~ms}$ post-offset of audio sentences by using a SR-Research EyeLink-1000. The sampling rate was $1000 \mathrm{~Hz}$. Participants sat $70 \mathrm{~cm}$ from the screen and they usually took about 1 hour to finish this experiment.

\section{Time windows}

Similar to Thothathiri and Snedeker (2008a), we analyzed eye-movements in the time window of NP1 (e.g., "Qiupai (racket)" or "Qiuyuan (football player)"). However, processing the 
verb (e.g., "huan (returns)") is enough for comprehenders to engage in prediction about structure (Arai et al., 2007). Therefore, we constructed two critical ambiguous time windows: the target verb window (1000ms) from 200ms (200ms after the onset of the target verb, following Matin et al., 1993; Thothathiri \& Snedeker, 2008a) to $1200 \mathrm{~ms}$ (200ms after the onset of the first syllable of NP1, "Qiu"); and the first syllable of NP1 window (550ms) from $1200 \mathrm{~ms}$ to $1750 \mathrm{~ms}$ (200ms after the onset of second syllable of NP1, "yuan" or "pai").

\section{Data analysis}

We recorded the fixation time within a rectangular region of interest around the corresponding entities (e.g., agent, recipient, theme) for the target trials. The corresponding visual item to the ambiguous noun phrase were the animate recipient (e.g., "Qiuyuan [football player]") and the inanimate theme (e.g., "Qiupai [racket]"). We then calculated the proportion of looks to these two items within the two ambiguous time windows, because looks to the recipient after verb onset indicated the prediction of DO structure, whereas looks to the theme indicated PO structure. We used the empirical logit transformation (Barr, 2008) to calculate the gaze probability data of recipient and theme. Then we computed the dependent variable as the difference score of gaze probability between recipient and theme (Thothathiri \& Snedeker, 2008a).

The two critical ambiguous time windows were analyzed with linear mixed models using the "Ime4" package (Bates \& Maechler, 2009) in R, with the main effect of two variables (verb bias and structure of primes) and their interaction effect as the predictors. Due to model convergence issues (Barr et al., 2013), we reduced the random effect structure with an intercept for subjects, and a slope of the interaction and intercept for items in the LME model for the target verb time window; and the random effect structure with a slope of structure and intercept for subjects, and a slope of verb bias and intercept for items in the LME model of the time window of the first syllable of NP1. In order to analyze the time course of the structure prediction within each time window, we used the method of cluster-based permutation analysis (Barr et al., 2014; Borovsky et al., 2016; Maris \& Oostenveld, 2007; Weighall et al., 2017). We implemented the permutation test with the "permuco" package in R (Frossard \& Renaud, 2019). We tested two main effects and one corresponding interaction effect of two factors (verb bias and structure) with a full random structure for subjects (by-subject analysis) or items (by-item analysis) in each time bin (50ms) when controlling the family-wise error rate. We used $F$ tests with a threshold of $p<.05$ 
(two sided), 5000 permutations, and the sum as a clustermass statistic, to detect when the structure prediction began and ended within the ambiguous time windows. Data and scripts are available online: https://osf.io/6kcyz/.

\section{Results}

\section{Traditional time-window analysis}

Figure 4A shows the time course of eye-movement data from the onset of the target verb during comprehension of target sentences after primes with different structures (DO vs. PO). We found a significant main effect of structure within both the time window of the target verb (e.g., 'huan') $(\beta=.52, S E=.18, t=2.96, p<.01)$ and the first syllable of NP1 (e.g., 'Qiu') $(\beta=.80$, $S E=.23, t=3.44, p<.001)$. The time course graph illustrates a clear priming effect with a larger proportion of looks to recipients after DO primes than PO primes in both ambiguous time windows. Figure 4B splits out the prime conditions for verb bias and suggests that bias modulates the priming effect. Indeed, the interaction between structure and verb bias was significant in the time window of the first syllable of NP1 $(\beta=-.95, S E=.45, t=-2.11, p<.05)$ (see Figure 5B), but it was not significant in the time window of the target verb $(\beta=-.22, S E=.37, t=-.60, p>.1)$ (see Figure $5 \mathrm{~A})$. There was no main effect of verb bias $(p>.1)$. Furthermore, in the time window of the first syllable of NP1 (see Figure 5B), the proportion of looks to the recipient was larger after PO-biased verbs followed by DO primes than PO primes $(\beta=1.27, S E=.32, t=3.94, p<.001)$. The similar priming effect did not occur in DO-biased verbs $(p>.1)$. The proportion of looks to the recipient was marginally larger after PO-biased DO primes than after DO-biased DO primes $(\beta=.58, S E=$ $.32, t=1.80, p=.07)$. There was no inverse preference priming effect in the PO primes $(p>.1)$.

\section{Cluster-based permutation analysis for eye-tracking data}

For the main effect of structure, the permutation analysis indicated two significant clusters in the target verb window: a cluster from 550ms $-850 \mathrm{~ms}$ (cluster-mass $=37.15, p<.05$, for a bysubject analysis, see Figure 4A) or from 550ms - 900ms, cluster-mass $=59.64, p<.05$, for a byitem analysis); and the other is from 950ms - 1200ms (cluster-mass $=35.36, p<.05$, for the bysubject analysis; cluster-mass $=31.34, p=.046$, for the by-item analysis). Additionally, the analysis showed one significant cluster in the time window of the first syllable of NP1 from $1200 \mathrm{~ms}-1750 \mathrm{~ms}$ (cluster-mass $=88.87, p<.01$, for the by-subject analysis) or from $1300 \mathrm{~ms}-$ $1750 \mathrm{~ms}$ (cluster-mass $=68.74, p<.01$ for the by-item analysis). For the interaction between 
structure and verb bias, the results only showed one significant cluster from $1550 \mathrm{~ms}-1750 \mathrm{~ms}$ (cluster-mass $=20.30, p<.05$, for the by-subject analysis, see Figure 4B; cluster-mass $=31.43, p$ $<.05$, for the by-item analysis) in the NP1 window. There was a small cluster (from $600 \mathrm{~ms}-650 \mathrm{~ms}$ in the by-item analysis, or from $500 \mathrm{~ms}-650 \mathrm{~ms}$ in the by-subject analysis) in the target verb window, but it was not significant $(p>.1)$. Importantly, the significant cluster of the interaction $(1550 \mathrm{~ms}-1750 \mathrm{~ms})$ showed a similar pattern as the whole time window (Figure 5B). Within this cluster, the priming effect occurred in PO-biased verbs $(\beta=1.44, S E=.35, t=4.09, p<.001)$ but not for DO-biased verbs $(p>.1)$, and the inverse preference priming also occurred in DO primes $(\beta=.79, S E=.36, t=2.22, p<.05)$ but not in PO-primes $(p>.1)$.

\section{Figure 4}

Difference in proportion of looks to recipient and theme for each time bin (50ms) from onset of target verb.

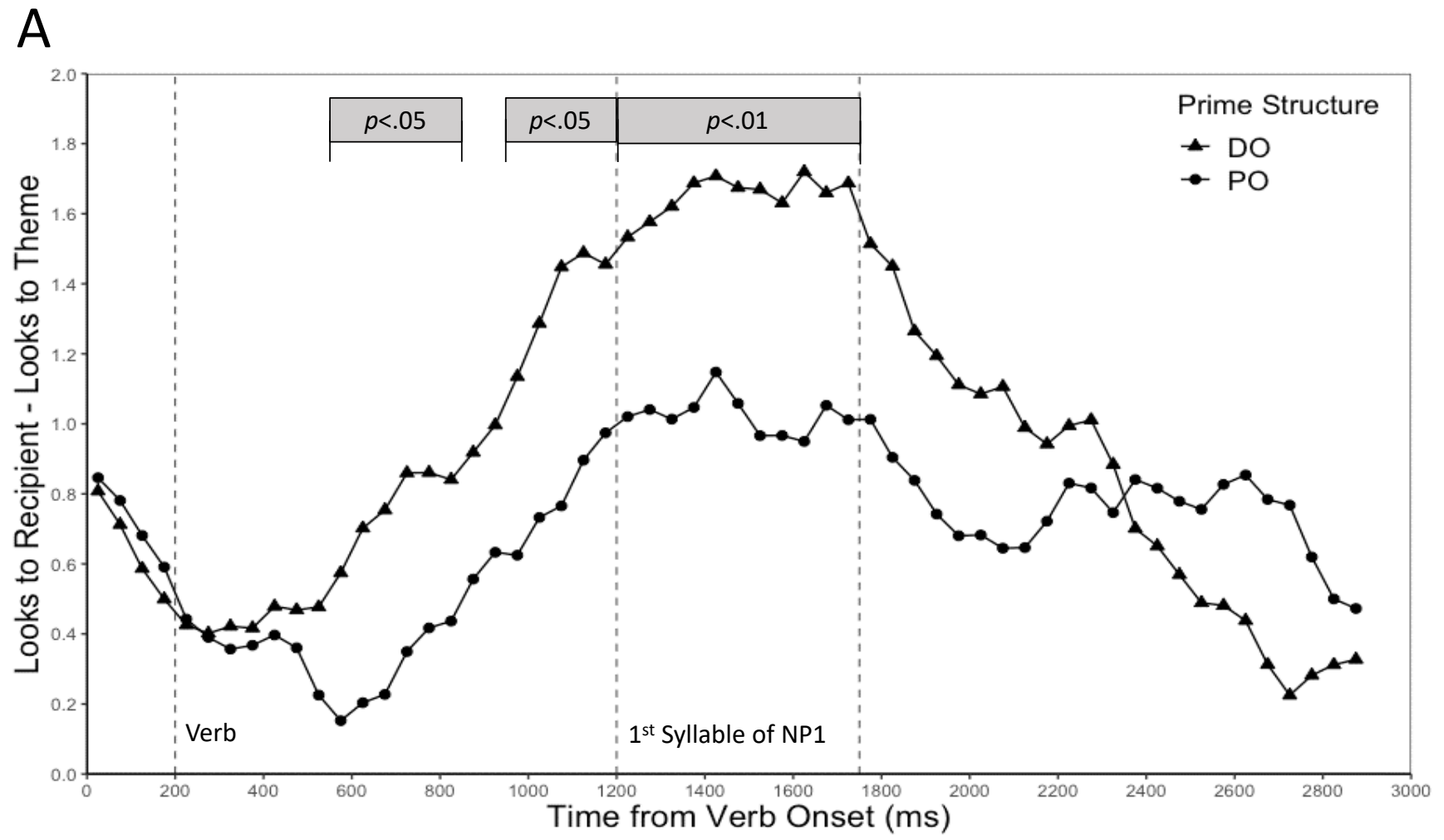




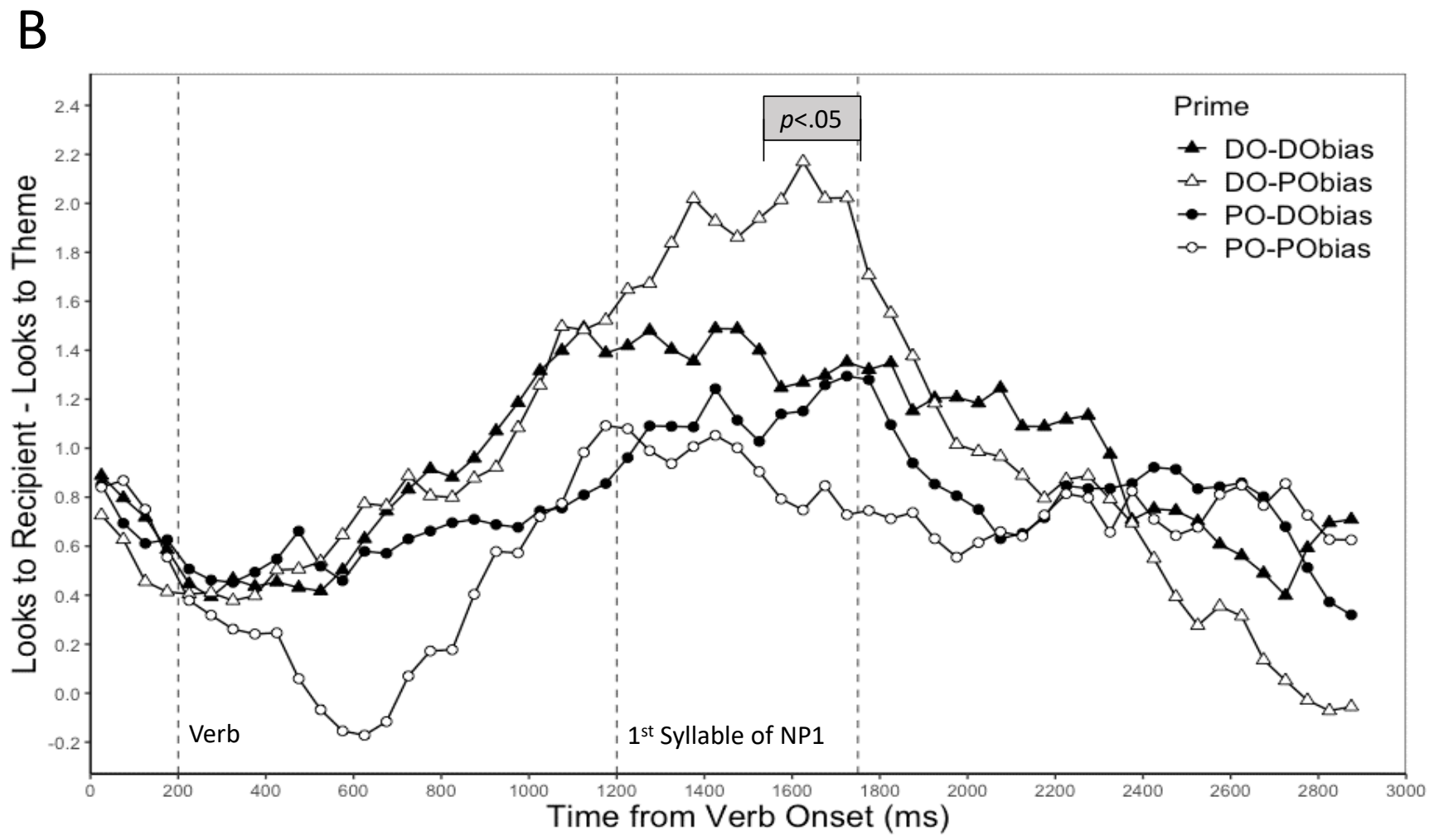

Note. The time window of verb is from $200 \mathrm{~ms}$ to $1200 \mathrm{~ms}$ and the time window of the first syllable of the first noun phrase is from $1200 \mathrm{~ms}$ to $1750 \mathrm{~ms}$. Plot A on the top indicates the difference in the proportions of looks to recipient (predicting DO structure) and to theme (predicting PO structure) after prime sentences with different structure (DO vs. PO); the grey rectangles within the time windows indicate the clusters where the main effect of structure was significant (by-subject analysis). Plot B on the bottom indicates the difference in the proportions of looks to recipient and theme after prime sentences with different structure (DO vs. PO) and prime verbs with different structure bias (DObias vs. PObias); the grey rectangles in the time window of the first syllable of the first noun phrase indicates the cluster where the interaction effect between structure and verb bias was significant (by-subject analysis).

\section{Figure 5}

Difference of gaze probability between recipient and theme for prime conditions with different structures (DO vs. PO) and verb bias (DObias vs. PObias) in two critical time windows. 
A

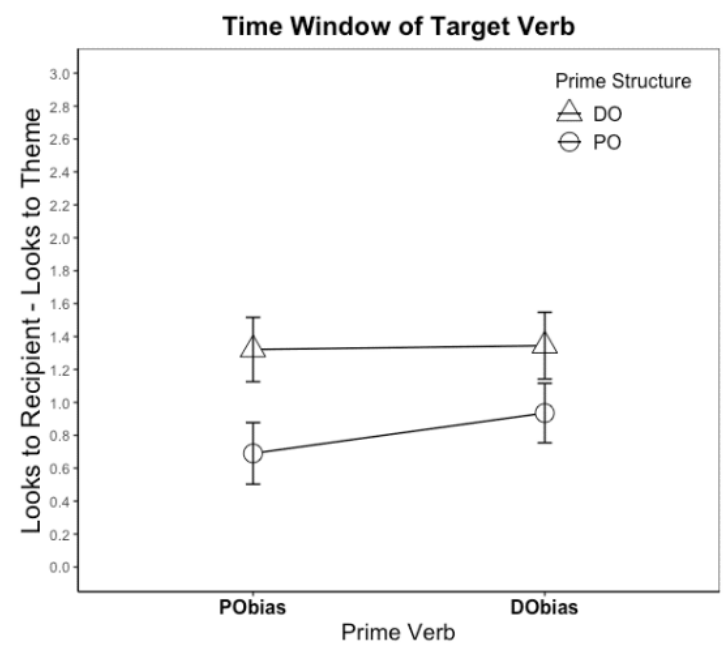

B

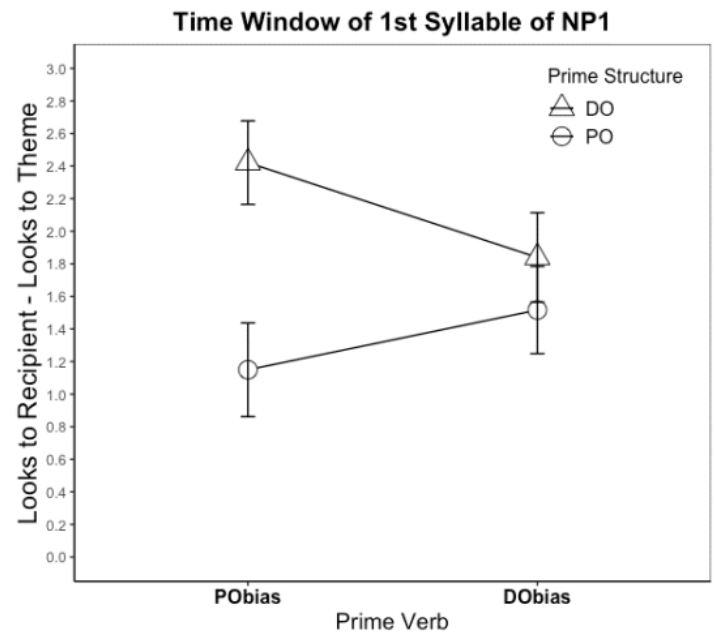

Note. Plot A on the left indicates the interaction between structure and verb bias in the time window of target verb $(p>1)$. Plot B on the right indicates the interaction between structure and verb bias in the time window of the first syllable of the first noun phrase $(p<.05)$. The error bars reflect standard errors from a by-participant analysis.

\section{Discussion}

This experiment demonstrated abstract structural priming in comprehension. Interestingly, the main effect of structure was already significant in the time window of the target verb, suggesting that the priming effect started soon after the onset of the target verb (see Figure 4A). As predicted by the implicit learning account, there was an inverse preference priming effect in Experiment 2 during the process of language comprehension, similar to that in production (Jaeger \& Snider, 2013). The interaction between structure and verb bias was significant in the time window of the first syllable of NP1 (see Figure 5B), consistent with Fine and Jaeger's (2013) reanalysis of Thothathiri and Snedeker's (2008a) comprehension study. This finding suggested that structure prediction in comprehension was modulated by the structure bias of the verbs. The inverse preference effect occurred with PO-biased prime verbs (i.e., primes with PO-biased verbs followed by the unexpected DO structure elicited more looks to recipients than when followed by the expected PO structure), but it did not occur in DO-biased verbs. One reason might be that the structure bias of PO-biased verbs is much larger than that of DO-biased verbs (i.e., -2.01 vs. 0.92), resulting in much larger prediction error for PO-biased verbs. This asymmetry is consistent with the results of a Dutch production study (Bernolet \& Hartsuiker, 2010). Dutch has a similar 
distribution of structure bias for ditransitives as Mandarin. Bernolet and Hartsuiker found that the strongest DO priming effect was induced by the strongest PO-biased verb (i.e., -1.63) and that the strength of the priming effect size linearly decreased with decreasing PO bias of the prime verb. In contrast, the PO priming effect with DO-biased verbs (i.e., 0.27) was much smaller than the DO priming effect with PO-biased verbs, and PO priming did not interact with the DO bias of the prime verb. Together, these findings suggested an error-based prediction system, in that larger prediction errors (related to the structure bias of the specific prime verbs) triggered a stronger priming effect.

\section{General Discussion}

In Experiment 1, we collected data from 367 Mandarin speakers to norm 48 dative verbs and found a strong PO bias overall. Then in Experiment 2, we manipulated the structure bias of prime verbs (verb bias) and prime structure in a visual-world eye-tracking study. First, we found stable structural priming in comprehension for Mandarin speakers even though the verbs were different between prime and target. In particular, comprehenders showed a higher proportion of looks to an animate recipient than to an inanimate theme when they heard the target sentence after a DO prime, and a higher proportion of looks to the inanimate theme than to the animate recipient after a PO prime. Structural priming occurred in both the time windows of the target verb and of the first syllable of the first noun phrase. Second, the structural priming effect was modulated by verb bias, so that structural priming was inversely related to the structure bias of prime verbs. This inverse preference effect occurred in the time window of the first syllable of the first noun phrase. Specifically, priming was stronger after a DO prime with a verb biased towards the alternative structure (i.e., PO-bias) than with a verb biased towards the same structure (DO-bias). However, the inverse preference effect seemed to depend on the strength of the bias, with weaker priming when there was a weak structure bias (i.e., DO-biased verbs).

The abstract structural priming suggested that the prediction for upcoming structure in the target sentences was shared with previously experienced prime sentences, supporting a lexically independent representation of structure in comprehension, as is the case for production. This result replicates Thothathiri and Snedeker's (2008a, 2008b) findings in comprehension. Different from Thothathiri and Snedeker's studies, we only presented one prime sentence before the target 
sentence and nevertheless found structural priming, indicating that one prime is enough to yield abstract structural priming in comprehension (as is also true of production). Our finding differs from Arai et al. (2007) who did not find abstract structural priming, even though we used the same comprehension task. Ziegler and Snedeker (2019) suggested that the absence or presence of abstract priming depends on task demands. They argued that the task Arai et al.'s used requires little need to continuously predict the structure of the target sentences, because once comprehenders have heard the agent and target verb, they can easily identify the two post-verbal arguments (e.g., the animate entity is likely to be the recipient and the inanimate entity the theme). Additionally, the participants had ample time to access the concepts of all arguments during the $1500 \mathrm{~ms}$ preview after target picture onset. Given these task characteristics, there would be no need to predict structure. In contrast to this task-demand hypothesis, our findings suggested that comprehenders are in fact continuously predicting structural information of the target sentences in this task. We propose another interpretation of the absence of abstract structural priming in Arai et al.'s study. These authors did not strictly control the structure bias of the prime and target verbs, which may have confounded any priming effects. If the target verbs are relatively strongly biased towards either the DO or PO, there is little room for priming effects (see Bernolet \& Hartsuiker, 2010; Coyle \& Kaschak, 2008).

Importantly, when we manipulated verb bias and prime structure together in comprehension, we found an inverse preference priming effect. This finding indicated that structure prediction in comprehension is sensitive to the prediction error (i.e., larger prediction error triggered a stronger priming effect), in line with the hypothesis from the implicit learning account (Chang et al., 2006). Our findings are consistent with Fine and Jaeger's (2013) results: these authors observed inverse preference priming when comprehenders processed the first of two prime sentences but not when they processed the second prime sentence, presumably because comprehenders rapidly adapted to the unexpected structure in the first prime and then were less surprised by such a structure in the second prime. Additionally, the inverse priming effect in comprehension we observed here was on the basis of prediction errors in a task where the prime sentences were read aloud, which generalized Thothathiri and Snedeker's findings in a task where the prime sentences were comprehended in the auditory modality. Similarly, the inverse preference priming effect also occurred in production regardless of whether the prime sentences were only produced or comprehended (Bernolet \& Hartsuiker, 2010; Jaeger \& Snider, 2013; Peter et al., 
2015). Together, these findings support a cross-modality error-based prediction system serving both production and comprehension.

There are several alternative accounts that interpret structural priming with respect to residual activation of syntactic representations (Pickering \& Branigan, 1998) or shared representations of information structure (Prat-Sala \& Branigan, 2000). However, these accounts cannot easily explain the inverse preference priming effects. According to Reitter et al. (2011), the residual activation model would predict a preference priming effect rather than an inverse preference effect, because the more strongly biased structure more strongly activates the structure representation in the processing of prime sentences, resulting in higher resting activation. In contrast, the information-structure account predicts an overall priming effect when the order of thematic roles are shared or the entity is emphasized (Griffin \& Weinstein-Tull, 2003), which would not be modulated by the structure bias of verbs. Together, our findings supported the prediction of implicit learning accounts, that "structural priming is a form of error-based implicit learning, with the same learning mechanism responsible for syntax acquisition and for priming" ( see Chang et al., 2006, pp. 245-246).

However, abstract structural priming in comprehension does not always seem to be accompanied with a verb bias effect. In Experiment 2, structural priming occurred in the time window of the target verb but the inverse preference priming did not. This finding cannot be explained by the implicit learning account, which predicts that priming effects would be tied to inverse preference priming, because both effects would occur as a result of weight changes in response to prediction error during prime sentence processing, leading to an altered bias for either the DO or PO. It is not obvious why part of this altered bias would surface in both time windows, but another part of the bias only during the later time window. One possibility might be that, in contrast to an implicit learning account, the inverse preference effect and the basic priming effect have different loci in the system. In the literature on structural priming in production, some authors have argued for a role of both implicit learning and explicit memory effects, even in abstract structural priming (e.g., Bernolet et al., 2016). A less interesting possibility, which cannot be ruled out, however, is that the magnitude of the inverse preference effect is simply much smaller than that of the basic priming effect. As a result, any such effect may have been strongly affected by noise in the data and remain undetected in the early time window of the target verb. One way to minimize the interference of noise in the early time window and to further investigate the 
possibility that both abstract priming and the inverse preference effect have the same time course, might be to investigate inverse preference in a language with a larger range of verb biases. For instance English, with both strongly DO-biased and PO-biased dative verbs, allows for the manipulation of strong verb bias mismatch conditions in both $\mathrm{DO}$ and PO prime sentences.

A final important finding is the generalization of abstract structural priming in comprehension to Mandarin. Because of the language properties of Mandarin (i.e., flexible word order and a lack of markers for morphophonology, see P. Li, 1996 and P. Li et al., 1993), many studies proposed a language-specific hypothesis for Mandarin, according to which semantic processing precedes syntactic processing in comprehension (Wang et al., 2013; Yang et al., 2009; Y. Zhang et al., 2010, 2013). These studies rarely showed an early sensitivity to violations of word category (e.g., ELAN effect), in contrast to studies in English. Instead, they showed difficulties with semantic integration (e.g., N400 effect; Zhou et al., 2009). However, we observed structure prediction effects for Mandarin comprehenders, even though in general they may have difficulties to predict syntactic information consistently. Why do the priming and EEG studies in Mandarin lead to different conclusions? One interpretation is that Mandarin comprehenders can predict structure information independently after they successfully access the concepts of the post-verbal arguments in the sentence (based on visual object recognition in the visual-world paradigm), but not when they have no access yet to the concepts (as many EEG studies did not find any early prediction effects of syntax in violation conditions of both semantics and syntax, see Wang et al., 2013). For future studies, it would therefore be interesting to investigate whether Mandarin comprehenders can predict structure information before they can access the concepts of postverbal arguments. In particular, can comprehenders still make a syntactic prediction when the semantic prediction can also help them to solve the ambiguity in language processing?

Our finding of an error-based structure prediction effect in Mandarin Chinese is consistent with the findings in some Indo-European languages (i.e., Dutch and English, see Bernolet \& Hartsuiker, 2010; Fine \& Jaeger, 2013; Jaeger \& Snider, 2013), suggesting a similar mechanism for different languages. In the view of implicit learning (Chang et al., 2006), speakers or comprehenders predict structure based on a shared representation of languages, which starts from their first language learning. Therefore, it suggests that inverse preference priming effects should occur between languages for multilinguals. Indeed, many studies of bilinguals and multilinguals demonstrated that priming effect occurs across languages (e.g., English, French, and Dutch in 
Hartsuiker, Beerts, Loncke, Desmet, \& Bernolet, 2016; and German and English in Kidd, Tennant, \& Nitschke, 2015; see Van Gompel \& Arai, 2018, for a recent review). But it remains to be seen whether this between-languages structural priming depends on an error-based prediction mechanism as in within-languages processing. On one scenario it should not, as a prediction error related to Language $\mathrm{X}$ is not informative about the distribution of structures in Language $\mathrm{Y}$. Therefore, an interesting research question for the future is whether the inverse preference priming effect occurs between different languages.

In conclusion, we performed a norming study to create a valuable resource for further studies: bias information for 48 dative verbs in Mandarin. In the following comprehension study, we found structural priming in the absence of verb repetition, suggesting that abstract structural representation is involved in comprehension as it is in production. Moreover, we found that the abstract priming was modulated by structural bias of prime verbs. This finding supports the hypothesis of an error-based prediction mechanism for structural priming, as proposed by the implicit learning theory.

\section{Acknowledgements}

This work was supported by a grant from Key Project of National Social Science Foundation of China (15AZD048). We thank the support from China Scholarship Council (CSC). Xuemei Chen was supported by China Scholarship Council (CSC).

\section{Reference}

Arai, M., van Gompel, R. P. G., \& Scheepers, C. (2007). Priming ditransitive structures in comprehension. Cognitive Psychology, 218-250. https://doi.org/10.1016/j.cogpsych.2006.07.001

Barr, D. J. (2008). Analyzing "visual world" eyetracking data using multilevel logistic regression. Journal of Memory and Language, 59(4), 457-474. https://doi.org/10.1016/j.jm1.2007.09.002

Barr, D. J., Jackson, L., \& Phillips, I. (2014). Using a voice to put a name to a face: The psycholinguistics of proper name comprehension. In Journal of Experimental Psychology: General (Vol. 143, Issue 1, pp. 404-413). https://doi.org/10.1037/a0031813

Barr, D. J., Levy, R. P., Scheepers, C., \& Tily, H. J. (2013). Random effects structure for 
confirmatory hypothesis testing: Keep it maximal. Journal of Memory and Language, 68(3), 255-278. https://doi.org/10.1016/j.jml.2012.11.001

Bates, D., \& Maechler, M. (2009). Package 'Ime4'(Version 0.999375-32): linear mixed-effects models using S4 classes. CRAN. R Foundation for Statistical Computing, Vienna, Austria. https://cran.r-project.org/package=lme4

Bernolet, S., Collina, S., \& Hartsuiker, R. J. (2016). The persistence of syntactic priming revisited. Journal of Memory and Language, 91, 99-116. https://doi.org/10.1016/j.jm1.2016.01.002

Bernolet, S., \& Hartsuiker, R. J. (2010). Does verb bias modulate syntactic priming? Cognition, 114(3), 455-461. https://doi.org/10.1016/j.cognition.2009.11.005

Bock, K. (1986a). Meaning, Sound, and Syntax. Lexical Priming in Sentence Production. Journal of Experimental Psychology: Learning, Memory, and Cognition, 12(4), 575-586. https://doi.org/10.1037/0278-7393.12.4.575

Bock, K. (1986b). Syntactic persistence in language production. Cognitive Psychology, 18, 355387.

Bock, K., \& Griffin, Z. M. (2000). The persistence of structural priming: Transient activation or implicit learning? Journal of Experimental Psychology: General, 129(2), 177-192. https://doi.org/10.1037/0096-3445.129.2.177

Borovsky, A., Ellis, E. M., Evans, J. L., \& Elman, J. L. (2016). Semantic Structure in Vocabulary Knowledge Interacts With Lexical and Sentence Processing in Infancy. Child Development, 87(6), 1893-1908. https://doi.org/10.1111/cdev.12554

Branigan, H. P., Pickering, M. J., \& McLean, J. F. (2005). Priming Prepositional-Phrase Attachment During Comprehension. Journal of Experimental Psychology: Learning, Memory, and Cognition, 31(3), 468-481.

Branigan, H. P., Pickering, M., Zhang, Q., Liu, S., Wang, S., \& Huang, J. (2018). Is syntactic prediction universal? Evidence from visual world syntactic priming in Mandarin sentence comprehension. AMLaP-Asia.

Bresnan, J., Cueni, A., Nikitina, T., \& Baayen, R. H. (2007). Predicting the dative alternation. In G. Bouma, I. Kraemer, \& J. Zwarts (Eds.), Cognitive foundations of interpretation (pp. 69- 
94). Amsterdam: KNAW.

Cai, Q., \& Brysbaert, M. (2010). SUBTLEX-CH: Chinese word and character frequencies based on film subtitles. PLoS ONE, 5(6). https://doi.org/10.1371/journal.pone.0010729

Cai, Z., Pickering, M. J., \& Branigan, H. P. (2012). Mapping concepts to syntax: Evidence from structural priming in Mandarin Chinese. Journal of Memory and Language, 66, 833-849.

Cai, Z., Pickering, M. J., Wang, R., \& Branigan, H. P. (2015). It is there whether you hear it or not: Syntactic representation of missing arguments. Cognition, 136, 255-267.

Cai, Z., Pickering, M. J., Yan, H., \& Branigan, H. P. (2011). Lexical and syntactic representations in closely related languages: Evidence from Cantonese-Mandarin bilinguals. Journal of Memory and Language, 65, 431-445.

Chang, F. (2000). Structural priming as implicit learning: A comparison of models of sentence production. Journal of Psycholinguistic Research, 29(2), 217-230. https://doi.org/10.1023/A:1005101313330

Chang, F., Dell, G. S., \& Bock, K. (2006). Becoming syntactic. Psychological Review, 113(2), 234-272. https://doi.org/10.1037/0033-295X.113.2.234

Chen, X., Branigan, H. P., Wang, S., Huang, J., \& Pickering, M. J. (2019). Syntactic representation is independent of semantics in Mandarin: evidence from syntactic priming. Language, Cognition and Neuroscience, $0(0), 1-10$. https://doi.org/10.1080/23273798.2019.1644355

Colleman, T. (2006). De Nederlandse datiefalternantie. Een constructioneel en corpusgebaseerd onderzoek[The Dutch data variance: a constructional and corpus-based study]. Ghent University.

Colleman, T. (2009). Verb disposition in argument structure alternations: a corpus study of the dative alternation in Dutch. Language Sciences, 31(5), 593-611. https://doi.org/10.1016/j.langsci.2008.01.001

Colleman, T., \& Bernolet, S. (2013). Alternation biases in corpora vs. picture description experiments: DO-biased and PD-biased verbs in the Dutch dative alternation. Frequency Effects in Language Representation, 87-126. https://doi.org/10.1515/9783110274073.87

Coyle, J. M., \& Kaschak, M. P. (2008). Patterns of experience with verbs affect long-term 
cumulative structural priming. Psychonomic Bulletin and Review, 15(5), 967-970. https://doi.org/10.3758/PBR.15.5.967

Delong, K. A., Troyer, M., \& Kutas, M. (2014). Pre-Processing in Sentence Comprehension: Sensitivity to Likely Upcoming Meaning and Structure. Language and Linguistics Compass, 8(12), 631-645. https://doi.org/10.1111/lnc3.12093

Fine, A. B., \& Jaeger, T. F. (2013). Evidence for Implicit Learning in Syntactic Comprehension. Cognitive Science, 37(3), 578-591. https://doi.org/10.1111/cogs.12022

Frossard, J., \& Renaud, O. (2019). Permutation tests for regression, ANOVA and comparison of signals: the permuco package. $R$ Package Version 1.1.0. https://cran.rproject.org/web/packages/permuco/vignettes/permuco_tutorial.pdf

Griffin, Z. M., \& Weinstein-Tull, J. (2003). Conceptual structure modulates structural priming in the production of complex sentences. Journal of Memory and Language, 49(4), 537-555. https://doi.org/10.1016/j.jml.2003.08.002

Hartsuiker, R. J., Beerts, S., Loncke, M., Desmet, T., \& Bernolet, S. (2016). Cross-linguistic structural priming in multilinguals: Further evidence for shared syntax. Journal of Memory and Language, 90, 14-30. https://doi.org/10.1016/j.jml.2016.03.003

Huang, J., Pickering, M. J., Chen, X., Cai, Z., Wang, S., \& Branigan, H. P. (2019). Does language similarity affect representational integration? Cognition, 185(March 2018), 83-90. https://doi.org/10.1016/j.cognition.2019.01.005

Huang, J., Pickering, M. J., Yang, J., Wang, S., \& Branigan, H. P. (2016). The independence of syntactic processing in Mandarin: Evidence from structural priming. Journal of Memory and Language, 91, 81-98. https://doi.org/10.1016/j.jml.2016.02.005

Huettig, F., Rommers, J., \& Meyer, A. S. (2011). Using the visual world paradigm to study language processing: A review and critical evaluation. Acta Psychologica, 137(2), 151-171. https://doi.org/10.1016/j.actpsy.2010.11.003

Jaeger, T. F., \& Snider, N. (2008). Implicit learning and syntactic persistence: Surprisal and Cumulativity. Proceedings of the 30th Annual Meeting of the Cognitive Science Society (CogSci08), January, 1061-1066. 
Jaeger, T. F., \& Snider, N. E. (2013). Alignment as a consequence of expectation adaptation: Syntactic priming is affected by the prime's prediction error given both prior and recent experience. Cognition, 127(1), 57-83. https://doi.org/10.1016/j.cognition.2012.10.013

Kidd, E., Tennant, E., \& Nitschke, S. (2015). Shared abstract representation of linguistic structure in bilingual sentence comprehension. Psychonomic Bulletin and Review, 22(4), 1062-1067. https://doi.org/10.3758/s13423-014-0775-2

Levy, R. (2008). Expectation-based syntactic comprehension. Cognition, 106(3), 1126-1177. https://doi.org/10.1016/j.cognition.2007.05.006

Li, C. N., \& Thompson, S. A. (1989). Mandarin Chinese: A functional reference grammar. Berkeley: University of California Press.

Li, P. (1996). The temporal structure of spoken sentence comprehension in Chinese. Perception and Psychophysics, 58(4), 571-586. https://doi.org/10.3758/BF03213091

Li, P., Bates, E., \& MacWhinney, B. (1993). Processing a language without inflections: A reaction time study of sentence interpretation in Chinese. Journal of Memory and Language, 32, 169192.

Mahowald, K., James, A., Futrell, R., \& Gibson, E. (2016). A meta-analysis of syntactic priming in language production. Journal of Memory and Language, 91, 5-27. https://doi.org/10.1016/j.jml.2016.03.009

Man, Z. (2003). Generative Grammar and the Double Object Construction in Chinese. Modern Foreign Languages(Quarterly), 26(3), 233-240.

Maris, E., \& Oostenveld, R. (2007). Nonparametric statistical testing of EEG- and MEG-data. Journal of Neuroscience Methods, 177-190. https://doi.org/10.1016/j.jneumeth.2007.03.024

Matin, E., Shao, K. C., \& Boff, K. R. (1993). Saccadic overhead: Information-processing time with and without saccades. Perception \& Psychophysics, 53(4), 372-380. https://doi.org/10.3758/BF03206780

Perek, F., \& Goldberg, A. E. (2015). Generalizing beyond the input: The functions of the constructions matter. Journal of Memory and Language, 84, 108-127. 
https://doi.org/10.1016/j.jml.2015.04.006

Perek, F., \& Goldberg, A. E. (2017). Linguistic generalization on the basis of function and constraints on the basis of statistical preemption. Cognition, 168, 276-293. https://doi.org/10.1016/j.cognition.2017.06.019

Peter, M. S., Chang, F., Pine, J. M., Blything, R., \& Rowland, C. F. (2015). When and how do children develop knowledge of verb argument structure? Evidence from verb bias effects in a structural priming task. Journal of Memory and Language, 81, 1-15. https://doi.org/10.1016/j.jml.2014.12.002

Pickering, M. J., \& Branigan, H. P. (1998). The Representation of Verbs: Evidence from Syntactic Priming in Language Production. Journal of Memory and Language, 39, 633-651.

Prat-Sala, M., \& Branigan, H. P. (2000). Discourse Constraints on Syntactic Processing in Language Production: A Cross-Linguistic Study in English and Spanish. Journal of Memory and Language, 42(2), 168-182. https://doi.org/10.1006/jmla.1999.2668

Reitter, D., Keller, F., \& Moore, J. D. (2011). A Computational Cognitive Model of Syntactic Priming. Cognitive Science, 35(4), 587-637. https://doi.org/10.1111/j.15516709.2010.01165.x

Tanenhaus, M. K., Spivey-Knowlton, M. J., Eberhard, K. M., \& Sedivy, J. C. (1995). Integration of visual and linguistic information in spoken language comprehension. Science, 268(5217), 1632-1634. https://doi.org/10.1126/science.7777863

Thothathiri, M., \& Braiuca, M. C. (2020). Distributional Learning in English: The Effect of VerbSpecific Biases and Verb-General Semantic Mappings on Sentence Production. Journal of Experimental Psychology: Learning Memory and Cognition. https://doi.org/10.1037/xlm0000814

Thothathiri, M., \& Rattinger, M. G. (2016). Acquiring and producing sentences: Whether learners use verb-specific or verb-general information depends on cue validity. Frontiers in Psychology, 7(MAR), 1-15. https://doi.org/10.3389/fpsyg.2016.00404

Thothathiri, M., \& Snedeker, J. (2008a). Give and take: Syntactic priming during spoken language comprehension. Cognition, 108(1), 51-68. https://doi.org/10.1016/j.cognition.2007.12.012 
Thothathiri, M., \& Snedeker, J. (2008b). Syntactic priming during language comprehension in three- and four-year-old children. Journal of Memory and Language, 58(2), 188-213. https://doi.org/10.1016/j.jml.2007.06.012

Traxler, M. J. (2008). Lexically independent priming in online sentence comprehension. Psychonomic Bulletin and Review, 15(1), 149-155. https://doi.org/10.3758/PBR.15.1.149

Van Gompel, R. P. G., \& Arai, M. (2018). Structural priming in bilinguals. Bilingualism, 21(3), 448-455. https://doi.org/10.1017/S1366728917000542

Wang, S., Mo, D., Xiang, M., Xu, R., \& Chen, H. C. (2013). The time course of semantic and syntactic processing in reading Chinese: Evidence from ERPs. Language and Cognitive Processes, 28(4), 577-596. https://doi.org/10.1080/01690965.2012.660169

Weighall, A. R., Henderson, L. M., Barr, D. J., Cairney, S. A., \& Gaskell, M. G. (2017). Eyetracking the time-course of novel word learning and lexical competition in adults and children. Brain and Language, 167, 13-27. https://doi.org/10.1016/j.bandl.2016.07.010

Yang, J., Wang, S., Chen, H. C., \& Rayner, K. (2009). The time course of semantic and syntactic processing in Chinese sentence comprehension: Evidence from eye movements. Memory and Cognition, 37(8), 1164-1176. https://doi.org/10.3758/MC.37.8.1164

Zhang, L. (1997). Shenme shi yiheyufa?[What is parataxis]. Hanyu Xuexi, 97, 58-61.

Zhang, W. (1991). "Ba zi jiegou" de yuyi jiqi yuyong fenxi [Semantics and Pragmatic Analysis of the 'BA' Structure]. Language Teaching and Research, 3, 88-103.

Zhang, Y., Li, P., Piao, Q., Liu, Y., Huang, Y., \& Shu, H. (2013). Syntax does not necessarily precede semantics in sentence processing: ERP evidence from Chinese. Brain \& Language, $126,8-19$.

Zhang, Y., Yu, J., \& Boland, J. E. (2010). Semantics Does Not Need a Processing License From Syntax in Reading Chinese. Journal of Experimental Psychology: Learning, Memory, and Cognition, 36(3), 765-781.

Zhou, X., Ye, Z., Cheung, H., \& Chen, H. C. (2009). Processing the Chinese language: An introduction. Language and Cognitive Processes, 24(7-8), 929-946. https://doi.org/10.1080/01690960903201281 
Ziegler, J., \& Snedeker, J. (2019). The use of syntax and information structure during language comprehension: Evidence from structural priming. Language, Cognition and Neuroscience, 34(3), 365-384. https://doi.org/10.1080/23273798.2018.1539757 